\title{
Tailoring force sensitivity and selectivity by microstructure engineering of multidirectional electronic skins
}

Jonghwa Park (1)', Jinyoung Kim', Jaehyung Hong², Hochan Lee', Youngoh Lee', Seungse Cho', Sung-Woo Kim³, Jae Joon Kim $\mathbb{B}^{3}$, Sung Youb Kim² and Hyunhyub Ko ${ }^{1}$

\begin{abstract}
Electronic skins (e-skins) with high sensitivity to multidirectional mechanical stimuli are crucial for healthcare monitoring devices, robotics, and wearable sensors. In this study, we present piezoresistive e-skins with tunable force sensitivity and selectivity to multidirectional forces through the engineered microstructure geometries (i.e., dome, pyramid, and pillar). Depending on the microstructure geometry, distinct variations in contact area and localized stress distribution are observed under different mechanical forces (i.e., normal, shear, stretching, and bending), which critically affect the force sensitivity, selectivity, response/relaxation time, and mechanical stability of e-skins. Microdome structures present the best force sensitivities for normal, tensile, and bending stresses. In particular, microdome structures exhibit extremely high pressure sensitivities over broad pressure ranges $\left(47,062 \mathrm{kPa}^{-1}\right.$ in the range of $<1 \mathrm{kPa}$, $90,657 \mathrm{kPa}^{-1}$ in the range of $1-10 \mathrm{kPa}$, and $30,214 \mathrm{kPa}^{-1}$ in the range of $10-26 \mathrm{kPa}$ ). On the other hand, for shear stress, micropillar structures exhibit the highest sensitivity. As proof-of-concept applications in healthcare monitoring devices, we show that our e-skins can precisely monitor acoustic waves, breathing, and human artery/carotid pulse pressures. Unveiling the relationship between the microstructure geometry of e-skins and their sensing capability would provide a platform for future development of high-performance microstructured e-skins.
\end{abstract}

\section{Introduction}

Recently, a large number of electronic skins with capabilities of detecting physical/chemical stimuli have been reported for applications in robotics, wearable electronics, and healthcare monitoring devices ${ }^{1-8}$. Owing to their wearable or body-attachable features, many types of electronic skins (e-skins) have been actively utilized as innovative tools for monitoring radial and carotid blood pressure $^{1,2}$, and electrophysiological signals ${ }^{3,4}$ and for

Correspondence: Hyunhyub Ko (hyunhko@unist.ac.kr)

${ }^{1}$ School of Energy and Chemical Engineering, Ulsan National Institute of

Science and Technology (UNIST), Ulsan Metropolitan City 689-798, Republic of Korea

${ }^{2}$ School of Mechanical and Nuclear Engineering, Ulsan National Institute of Science and Technology (UNIST), Ulsan Metropolitan City 689-798, Republic of Korea

Full list of author information is available at the end of the article.

These authors contributed equally: Jonghwa Park, Jinyoung Kim, Jaehyung Hong oximetry $^{5}$, as well as drug delivery ${ }^{6}$. For accurate and reliable monitoring of those signals, e-skins with enhanced sensitivity, selectivity, response time, and mechanical durability are required. In accordance with these demands, eskins with various bio-inspired micro/nanostructures (e.g., interlocking, hierarchical, crack, whisker, and fingerprint) and $2 \mathrm{D}$ or $3 \mathrm{D}$ shapes (e.g., serpentine, wrinkle, pyramid, dome, and porous) have been reported aiming to obtain high-performance and multifunctional e-skins ${ }^{9}$. In particular, owing to their effective geometrical advantages, regular arrays having various microstructures (e.g., hemisphere, pyramid, and pillar) have been used to improve the performance of e-skins based on different operation modes (e.g., resistive, capacitive, piezoelectric, and triboelectric). Specifically, dome-shaped or hemisphere-shaped microstructures have been reported to induce a large change in contact area and localized stress concentration, which are

\section{(c) The Author(s) 2018}

(c) (i) Open Access This article is licensed under a Creative Commons Attribution 4.0 International License, which permits use, sharing, adaptation, distribution and reproduction cc) in any medium or format, as long as you give appropriate credit to the original author(s) and the source, provide a link to the Creative Commons license, and indicate if changes were made. The images or other third party material in this article are included in the article's Creative Commons license, unless indicated otherwise in a credit line to the material. If material is not included in the article's Creative Commons license and your intended use is not permitted by statutory regulation or exceeds the permitted use, you will need to obtain permission directly from the copyright holder. To view a copy of this license, visit http://creativecommons.org/licenses/by/4.0/. 
advantageous for fabricating high-performance piezoresistive sensors ${ }^{10}$ and triggering a large amount of triboelectric charge $^{11}$ and a high piezopotential in the piezoelectric devices ${ }^{12}$. On the other hand, a large variation in local strain and compressibility of pyramid-shaped microstructures enhance force sensitivity and expand the dynamic sensing range of e-skins ${ }^{13-15}$. Inspired by the hierarchical structures in nature, such as insect legs, gecko foots, and beetle wings, pillar-shaped microstructures have been reported to provide selective and directional force-sensing properties, as well as stress-confinement effects $^{16-19}$. In addition, the strong adhesion properties of pillar structures provide a new possibility for skinattachable and wearable healthcare devices ${ }^{1}$.

Considering these geometrical effects of microstructure arrays on the performance of e-skins, previously, geometrical parameters such as shape, size, and space of microstructure arrays have been controlled to enhance the mechanical sensitivity and operation range of piezoresistive $^{20}$ and capacitive e-skins ${ }^{15}$ and the power generation of self-powered e-skins ${ }^{16-18,21-23}$. Since the geometrical shape of microstructure significantly affects the contact area and localized stress of microstructure under pressure, several attempts to find the relationship between the shape of microstructure and the sensitivity of various eskins have been performed. For example, Fan et al. controlled the surface of triboelectric sensors with different shapes of microstructure ${ }^{22}$. Lee et al. Investigated the structural shape effect on the formation of piezoelectric potential to optimize sensor performances ${ }^{23}$. Deng et al. studied the dependence of interfacial shape of microstructures (cuboid, cylinder, and pyramid) on the sensitivity of capacitive sensors ${ }^{24}$. Although previous e-skins having different microstructures have successfully shown improved tactile sensing performances, specific to some of desired applications, there have been no systematic studies of the effects of microstructure geometry on force-induced microstructure-deformation and the resulting force sensitivity and selectivity in response to multidirectional mechanical stimuli. In particular, the sensing performance of piezoresistive e-skins with microstructure arrays critically depends on the localized stress distribution of microstructures and the resulting contact area change when external mechanical forces are applied in different directions. Although the interlocked microdome structures have been reported to exhibit high pressure sensitivity ${ }^{25,26}$, indepth investigation of the effects of microstructures on the multidirectional sensing performances has not been done. A systematic and in-depth analysis of geometrical effects on sensing performance is important to enable the design of tunable e-skins having high force sensitivity and selectivity, which can be customized for diverse applications.

In this study, multidirectional force sensitive e-skins are shown to possess customizable force sensitivity and selectivity by controlling the specific microstructure geometry. To investigate the underlying relationship between microstructure geometry and force sensing capability, three kinds of piezoresistive sensors were fabricated based on carbon nanotube/elastomer composites having different surface microstructures (e.g., dome, pyramid, and pillar). To facilitate comparison of the geometrical shape effect on the stress sensing properties, an interlocked geometry of the different microstructure arrays was employed. This has been reported to enhance the variation in contact area and localized stress in the microstructures, thereby improving the stress sensitivity and multidirectional force sensing capabilities of $\mathrm{e}$ skins $^{25-28}$. For the fundamental understanding of the sensing mechanisms, experimental piezoresistive properties were compared to the finite-element simulation of contact-area change and the localized stress distribution of microstructured e-skins. It was found that piezoresistive e-skins having different microstructures exhibit distinct force sensitivity and selectivity mainly due to the specific variation in contact area, which depended on the geometrical shape of the microstructures, in response to multidirectional forces (e.g., normal, shear, tensile, and bending). As a result, microdome structures presented the best force sensitivities for normal, tensile, and bending stresses. On the other hand, for shear stress, micropillar structures exhibited the highest sensitivity. As proof-ofconcept demonstrations for potential applications in wearable healthcare devices, highly sensitive pressure sensors were used for detecting various bio-signals, including sound, human breath, and artery/carotid pulse pressures.

\section{Materials and methods \\ Fabrication of conductive composites with interlocked microstructures}

The synthesis of conductive composites was initiated by dispersion $(5 \mathrm{mg} / \mathrm{ml})$ of multi-walled carbon nanotubes (Sigma-Aldrich, $110-190 \mathrm{~nm}$ diameter and 5-9 $\mu \mathrm{m}$ length) in chloroform (Samchun, Korea). After $6 \mathrm{~h}$ of sonication, well-dispersed MWNTs solution was mixed with PDMS base (Sylgard 184, Dow Corning) with an $8 \mathrm{wt} \%$ ratio of MWNTs to PDMS. Subsequently, the MWNT/PDMS solution was mixed with a vortex and dried at $90{ }^{\circ} \mathrm{C}$ for $12 \mathrm{~h}$, in order to completely evaporate the chloroform. Prior to the micromolding process, the MWNT/PDMS precursor was combined with a curing agent (PDMS base: curing agent 10:1) and hexane ( $1 \mathrm{ml}$ per $0.5 \mathrm{~g}$ of PDMS) were added for reducing the viscosity of the dried precursor. The prepared MWNT/PDMS composite solution was poured into silicon molds with different micropatterns (i.e., dome, pyramid, and micropillar), followed by air bubble removal and thermal treatment at $90^{\circ} \mathrm{C}$ for $4 \mathrm{~h}$, 
resulting in the preparation of micropatterned MWNT/ PDMS composite films.

Silicon molds with micropattern arrays were fabricated via photolithography and etching processes. For manufacturing microdome-patterned and micropillarpatterned PDMS molds, hexagonally patterned photomasks (diameter and pitch: $3 \mu \mathrm{m}$ and $15 \mu \mathrm{m}$ for the microdome structure and $10 \mu \mathrm{m}$ and $15 \mu \mathrm{m}$ for the micropillar structure, respectively) and a positive photoresist (PR, AZ 5214E) were used for photolithography (mask aligner, MA/BA-6-8, Succ Microtec) on bare silicon wafers. To conduct the dry-etching process, a reverse patterning process was performed by deposition of a $50 \mathrm{~nm}$-thick chrome layer using the thermal evaporation technique and lift-off process of PR using acetone. The chrome layer acted as a hard mask during the dry-etching process. Each chrome-patterned silicon wafer was isotropically or anisotropically etched via reactive ion etching (RIE) or deep reactive ion etching (DRIE) processes, respectively. Finally, each silicon mold for fabricating microdome-patterned and micropillar-patterned PDMS was prepared by removing the chrome layer through wetetching and coating a self-assembled monolayer (SAM) on each silicon wafer to ease the PDMS-unmolding process. For the micropyramid-patterned PDMS mold, negative PR (AZ nLOF 2035) was patterned on a $\mathrm{SiO}_{2} / \mathrm{Si}$ wafer $\left(\mathrm{SiO}_{2}\right.$ layer thickness of $\left.50 \mathrm{~nm}\right)$ via photolithography employing a hexagonally patterned photomask with a $10 \mu \mathrm{m}$ diameter and $15 \mu \mathrm{m}$ pitch. The exposed $\mathrm{SiO}_{2}$ was then etched via a RIE process and PR was removed with acetone. The remaining $\mathrm{SiO}_{2}$ acted as a hard mask in the crystallographic wet-etching process of silicon, which was conducted by immersing it in $30 \mathrm{wt} \%$ potassium hydroxide for $10 \mathrm{~min}$ at $70{ }^{\circ} \mathrm{C}$. Next, the $\mathrm{SiO}_{2}$ mask was completely removed by wet-etching using $4 \%$ hydrofluoric acid. After SAM-coating this silicon wafer, the fabrication process was completed.

\section{Characterizations}

The geometrical information at the surface of micropatterned composite films was obtained using fieldemission SEM (FE-SEM; S-4800, Hitachi). Surface conductivity of the micro-patterned composites having different fabricating conditions was measured using the four-probe method with a semiconductor analyzer (4200SCS, Keithley). In addition, the mechanical modulus of the composite films was obtained using a tensile strain tester (Universal Testing Machine, WL2100, WITHLAB Co., Korea) with a strain rate of $500 \mathrm{~mm} / \mathrm{min}$.

\section{Measurements of the multifunctional sensing performances of the e-skins}

To measure the electrical properties of the e-skins under mechanical forces, a copper electrode is attached uniformly onto the fabricated composite film with silver paste and then thermally annealed at $90^{\circ} \mathrm{C}$ for $30 \mathrm{~min}$ to minimize the electrical loss at the composite-electrode interface. Next, both composite films were placed facing each other and connected to the electrical measurement systems. To measure the electrical property of e-skins in response to tensile and bending stress, copper electrodes are attached at the side of composite films and polyimide tapes were sued to fasten the electrode on the e-skins. The electrical properties of e-skins that depend on mechanical forces were measured using the two-probe method of the semiconductor analyzer (4200-SCS, Keithley) with an applied voltage of $1-5 \mathrm{~V}$. To precisely apply the mechanical forces, a pushing tester (JIPT-100, Junil Tech) was used for normal pressure, and a tensile/ bending test machine (JIPT-200, Junil Tech) was used to apply stretching/bending forces. Shear force was applied with a microstage system (Micro Motion Technology, Korea). In order to evaluate the capability of sound detection, e-skins were exposed to acoustic waves with a $100 \mathrm{~Hz}$ frequency from a speaker (SMS-A490, Samsung) at a constant distance of $1 \mathrm{~cm}$ and then electrical resistance signals were collected. For the healthcare signal detection, $1 \mathrm{~cm}^{2}$ e-skins were covered with polyimide film and bandaged onto the wrist, neck, and below nose, and the change in electrical signals was recorded to monitor artery and carotid pulse pressures and human breath. To show the possibility of integration with commercially available chip, e-skin was connected to the commercial chip (STM32F030C6T6, STMicroelectronics) and signals are detected by oscilloscope (DPO2022B, Tektronix).

\section{Finite-element method}

In order to calculate the contact area and localized stress of microstructured e-skins, structural analysis was conducted using finite-element simulations. Linear elastic materials with experimentally measured mechanical properties followed composite models with different microstructures. For the basic model for numerical analysis of contact area, more than 0.5 million linear tetrahedral and wedge elements (element size: 0.2 (Min.) and 2 (Max.)) with an adaptive mesh-refinement scheme were employed. Each element in case of interlocked microdome, micropyramid, and micropillar has element/ volume ratio of 31.8, 55.4, 73.0 and average aspect ratio of $1.54,1.57,1.56$, respectively. Multidirectional loading conditions were modulated by displacement controls and converted to the corresponding normal, shear, tensile, and bending forces forms. Based on the surface-to-surface contact scheme between two deformable bodies, mechanical contact, localized stress, and thickness of the composite can be obtained under proper loading conditions. 


\section{Results and discussion}

Electronic skins having interlocked microstructures and different geometries

Figure 1a shows a schematic representation of a piezoresistive e-skin constituting multi-walled carbon nanotubes/polydimethylsiloxane (MWNT/PDMS) composite films having interlocked microstructure arrays. In this case, the piezoresistive properties of the e-skin depend mainly on the bulk film resistance $\left(R_{\mathrm{F}}\right)$ and the contact resistance $\left(R_{\mathrm{C}}\right)$ between the two neighboring microstructures of conductive composite films (Fig. 1b). While the piezoresistance of conventional e-skins constituting a planar composite film is affected predominantly by the $R_{\mathrm{F}}$ change, the piezoresistance of the interlocked microstructures is dominated by variations in $R_{\mathrm{C}}$. For conductive composites, the piezoresistance is affected mainly by the tunneling resistance ${ }^{29,30}$. Similarly, in the interlocked MWNT/PDMS composite films of the

a

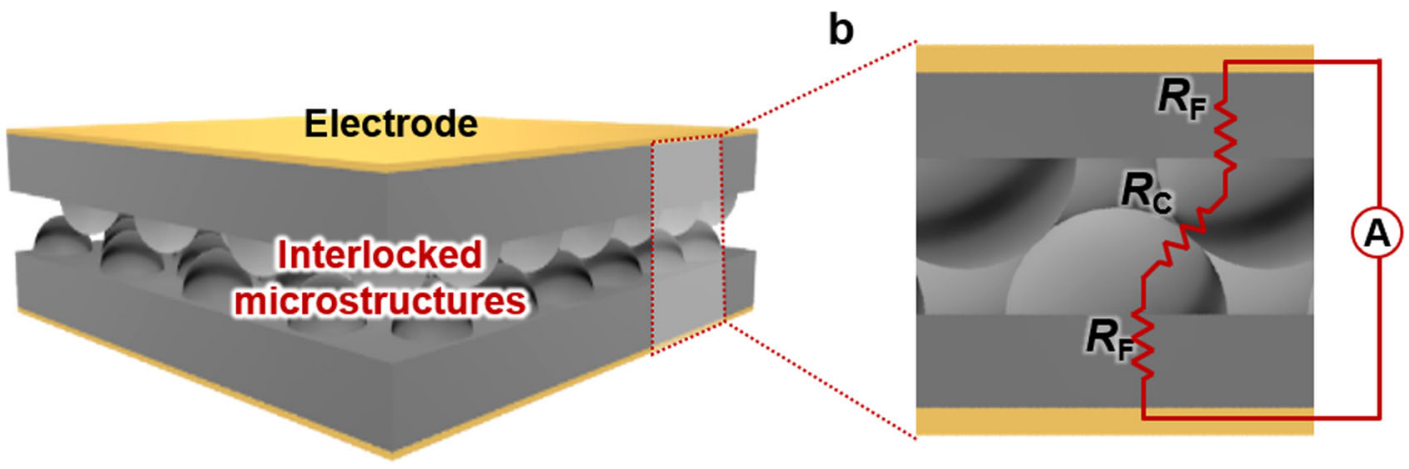

C
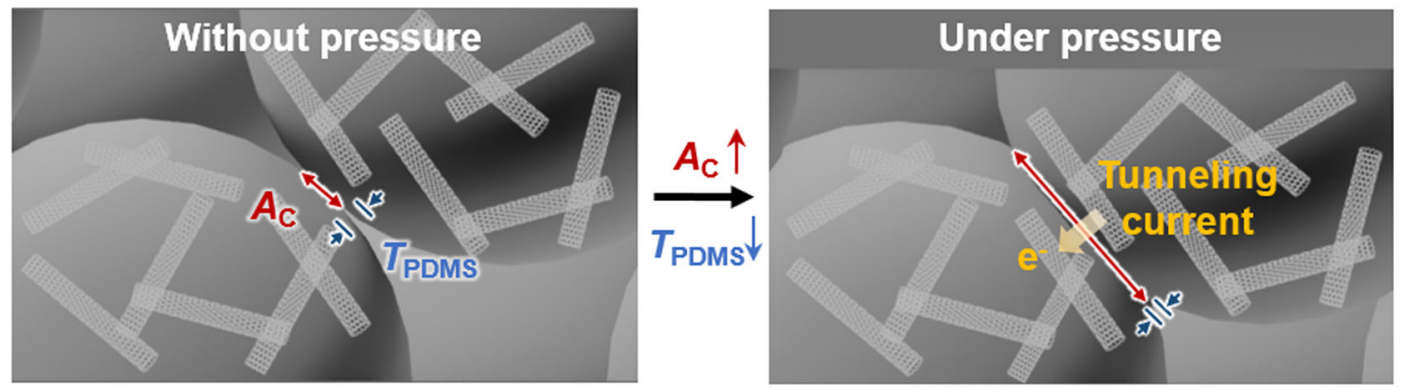

d

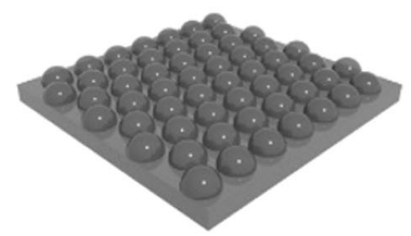

Microdome

e

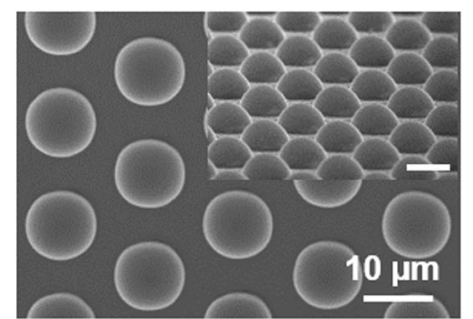

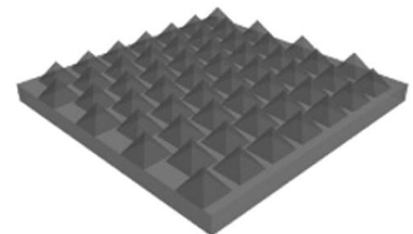

Micropyramid

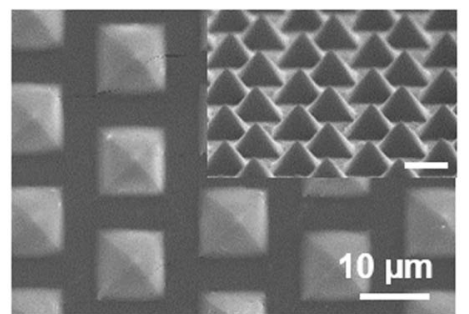

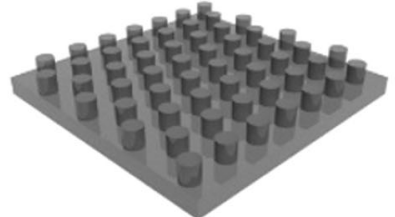

Micropillar

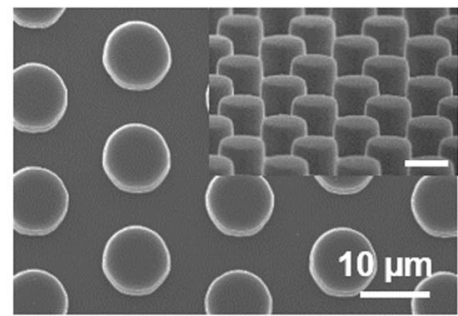

Fig. 1 Piezoresistive e-skins with interlocked microstructures having different surface morphologies. a Schematic illustration of the main structure of the e-skins with interlocked microstructures. $\mathbf{b}$ Schematic showing the bulk film resistance $\left(R_{\mathrm{F}}\right)$ and the contact resistance $\left(R_{C}\right)$ between the two neighboring microstructured films. c Pressure-sensing mechanism of piezoresistive e-skins having interlocked microstructures. $\mathbf{d}$ Schematic illustration of conductive composites with different microstructured arrays. e Top and tilted (inset) SEM images of the microstructured composites with different surface morphologies (microdome, micropyramid, and micropillar) 
present work, contact resistance is dominated by the tunneling resistance $\left(R_{\mathrm{T}}\right)$ between microstructured composite films, which is inversely proportional to the variation in contact area according to the equation $R_{\mathrm{T}}=(V / J) \times\left(1 / A_{\mathrm{CNT}}\right)$, where $V$ is the applied voltage, $J$ is the current density, and $A_{\mathrm{CNT}}$ is the electrical contact area between MWNTs, which is in turn proportional to the physical contact area $\left(A_{\mathrm{C}}\right)$ between interlocked microstructures $^{25}$. Therefore, the contact-area change critically affects contact resistance. In addition, the tunneling current $(J)$ through a thin PDMS insulating layer at the interface of interlocked microstructures exponentially increases with decreasing insulating layer thickness $\left(T_{\mathrm{PDMS}}\right)^{31}$. As schematically shown in Fig. 1c, the pressure sensing mechanism can be explained as follows. When an external pressure is applied on interlocked microdomes, stress accumulates at the tips of dome structures, and this concentrated stress can easily deform the microdomes and thus increase $A_{\mathrm{C}}$ between interlocked microstructures and decrease $T_{\mathrm{PDMS}}$ of the PDMS insulating layer between CNTs. These changes in contact area and barrier thickness significantly affect the tunneling currents through the thin PDMS insulating layer, causing a drastic increase in relative-current change as a response to pressure loading.

To investigate the effects of microstructure shape on piezoresistance, e-skins having three different surface morphologies (i.e., dome, pyramid, and pillar) were fabricated using the micromolding process (Fig. 1d, e). For this process, silicon replica molds with different micropattern arrays were produced via photolithography and etching processes (the detailed methodology is in the Experimental section). The fabricated microstructures had equal feature sizes; $10 \mu \mathrm{m}$ diameter, $15 \mu \mathrm{m}$ pitch, and $6 \mu \mathrm{m}$ height (Scanning electron microscope (SEM) images in Fig. 1e). For interlocked composite microstructures, high surface conductivity is preferred for enhancing pressure sensitivity due to the drastic $R_{\mathrm{C}}$ change with changing contact area under proper loading pressure ${ }^{25}$. In this study, interlocked composite microstructures having $8 \mathrm{wt} \%$ CNTs were utilized. For the different microstructure composites, sheet resistance exhibited similar values, around $1 \times 10^{4} \mathrm{ohm} / \mathrm{sq}$ (Supplementary Fig. S1).

\section{Multidirectional force-sensing capabilities of microstructured e-skins \\ Pressure-sensing capability of single microstructured e- skins}

Before discussing the structural effect of piezoresistance on interlocked CNT/PDMS composite microstructures, the pressure-sensing properties of single-sided composite films having different surface microstructures was investigated. Single-sided microstructured films have been widely employed to enhance the performance of flexible pressure sensors $^{10,14,17,32}$ and triboelectric generators ${ }^{11,22}$. For pressure-sensitive piezoresistance, a flat indium tin oxide (ITO) film was used as the top electrode on the surface of micropatterned composite films (Fig. 2a). In this case, the output current depends on the $R_{\mathrm{F}}$ of the bulk film and the $R_{\mathrm{C}}$ between the microstructure and electrode. The relative electrical current change $\left(\Delta I / I_{0}\right)$ as a function of normal pressure applied on top of the ITO electrode increased with increasing applied pressure (Fig. $2 \mathrm{~b}$ ). The $\Delta I / I_{0}$ values of single microstructured films increased as a function of pressure more rapidly in comparison to that of single planar structure. For quantitative analysis, the pressure sensitivity $(S)$ can be calculated using the equation $S=(\Delta I /$ $\left.I_{0}\right) / \Delta P$, where $I_{0}$ is the initial current, $\Delta I$ is the change in current after pressure loading, and $\Delta \mathrm{P}$ is the change in normal pressure. The linear pressure sensitivity exhibited different values depending on the microstructure shape (18.3, 12.6, and $5.3 \mathrm{kPa}^{-1}$ for microdome, micropyramid, and micropillar structures, respectively), all of which were much higher than that of a planar structure $\left(0.5 \mathrm{kPa}^{-1}\right)$ in the low-pressure range ( $<1 \mathrm{kPa})$ (Fig. $2 \mathrm{~b}$ and Supplementary Fig. S2a). As will be discussed later, the enhanced sensitivity of microstructured films, compared to the planar one, can be attributed to stress concentration at the small contact spot between the microstructure and ITO electrode, which resulted in an increase in contact area and decrease in PDMS barrier thickness, thereby increasing the tunneling current between the microstructure and electrode. For the planar structure, the external pressure mainly induced a change in inter-tube distance within the bulk composite film without affecting the contact area much, resulting in the lower pressure sensitivity than those of microstructures. Compared to the low pressure range, in the medium pressure range $(1-10 \mathrm{kPa})$, the pressure sensitivity decreased: $10.3,3.3,0.4$, and $0.2 \mathrm{kPa}^{-1}$ for microdome, micropyramid, micropillar, and planar structures, respectively (Fig. 2b and Supplementary Fig. S2b), but the effect of microstructure on the sensitivity did not change; sensitivity showed the same tendency as that in the lowpressure range (microdome $>$ micropyramid $>$ micropillar $>$ planar).

To further understand the piezoresistance of single-sided composite microstructures, finite-element simulations of contact-area variation of the microstructures under normal pressure were performed. When a pressure of $10 \mathrm{kPa}$ was applied on the composite films, microdome and micropyramid structures exhibited a local stress-concentration at the small contact spots, which was not observed for micropillar structures that do not have those small contact spots (Fig. 2c). In addition to the local stress-accumulation, the applied pressure induced a contact-area change, where dome and pyramid structures exhibited a significantly larger change than planar structures (Fig. 2d). As compared to pillar structures, the significantly smaller initial contact 
a

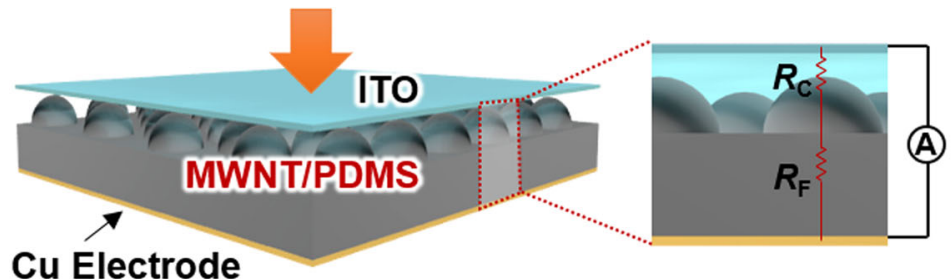

Cu Electrode
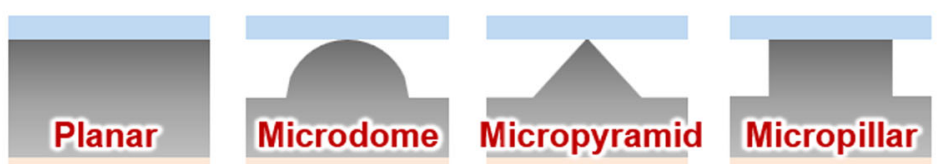

C

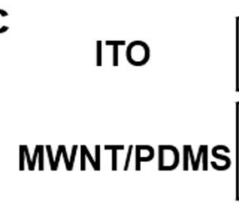

Stress (kPa)

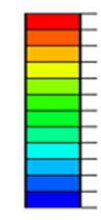

916

500

83
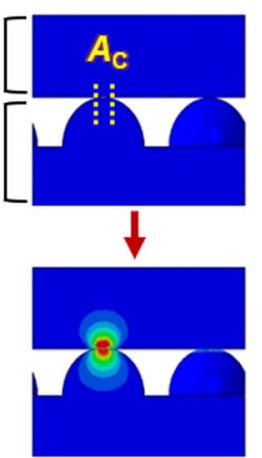

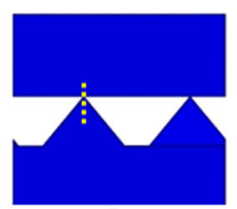

$10 \mathrm{kPa}$

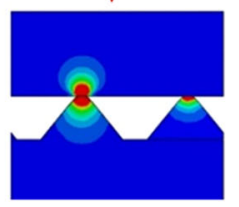

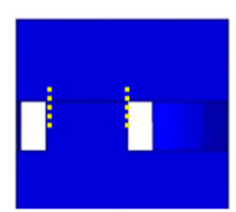

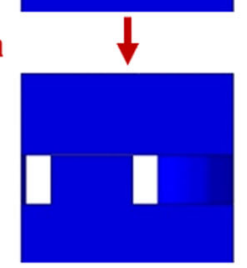

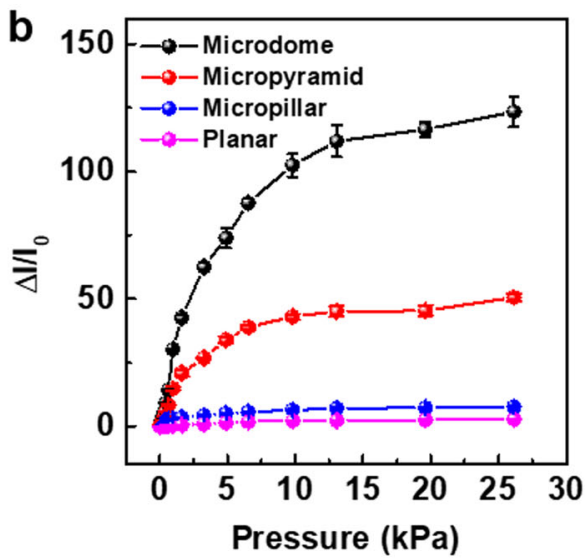

d

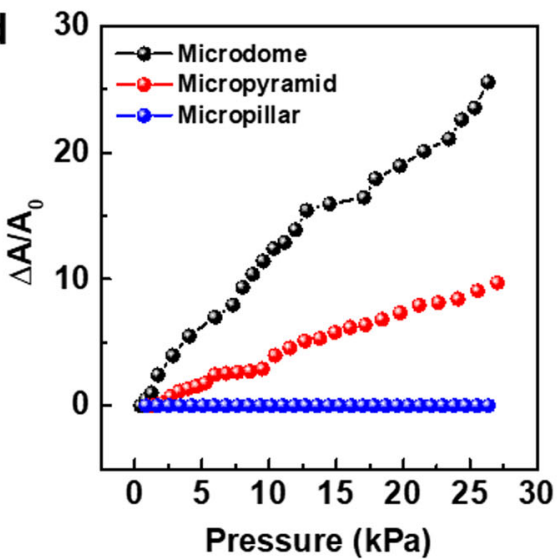

Fig. 2 Pressure-sensing capability of single microstructured e-skins. a Schematic illustration of the pressure-sensing principle of single microstructured e-skins with different surface morphologies. b Relative current changes in response to normal pressure. c Finite-element analysis (FEA) of localized stress distributions in response to pressure for different surface microstructure arrays (top layer: ITO, bottom layer: MWNT/PDMS composite). d Calculated relative contact-area changes in response to normal pressure

area of dome and pyramid structures under zero pressure led to a greater change in contact area under mechanical pressure (Supplementary Fig. S3), which resulted in a drastic increase in output current (Supplementary Fig. S4). In addition to the contact area, the concentrated stress can decrease the thickness of the PDMS dielectric barrier between the CNT and electrode, which is known to induce an exponential increase in tunneling current ${ }^{31}$. The change of microstructure thickness in response to pressure (Supplementary Fig. S5) implies that the barrier thickness decreases with increasing pressure. These changes in contact area and barrier thickness significantly increased the tunneling currents through the interface between the electrode and CNT composite as a response to pressure loading.

\section{Pressure-sensing capability of interlocked microstructured e-skins}

Considering the pressure-sensing capabilities of single-sided microstructured composites, interlocked composites having double microstructured surfaces are expected to further enhance pressure sensitivity owing to larger pressure-induced deformation of interlocked microstructures. Figure 3a shows the change in output current of CNT/PDMS composites having planar and interlocked microstructures under normal pressure. The interlocked composites with microdome, micropyramid, and micropillar arrays exhibited abrupt increases in current on application of a pressure up to $26 \mathrm{kPa}$, corresponding to $\Delta I / I_{0}$ values of approximately $1.38 \times 10^{6}$, $4.05 \times 10^{5}$, and $3.91 \times 10^{3}$, respectively, which are significantly higher than those of single microstructured films $\left(\Delta I / I_{0}<130\right)$. This behavior can be attributed mainly to the larger initial thickness of the insulating layer for interlocked microstructures, which resulted in a significantly lower initial current at the low-pressure range (Supplementary Fig. S6) and thus higher $\Delta I / I_{0}$ values compared to single microstructured films (Supplementary Fig. S4). It is worth noting that the interlocked microdome structures exhibited the largest change in current in 


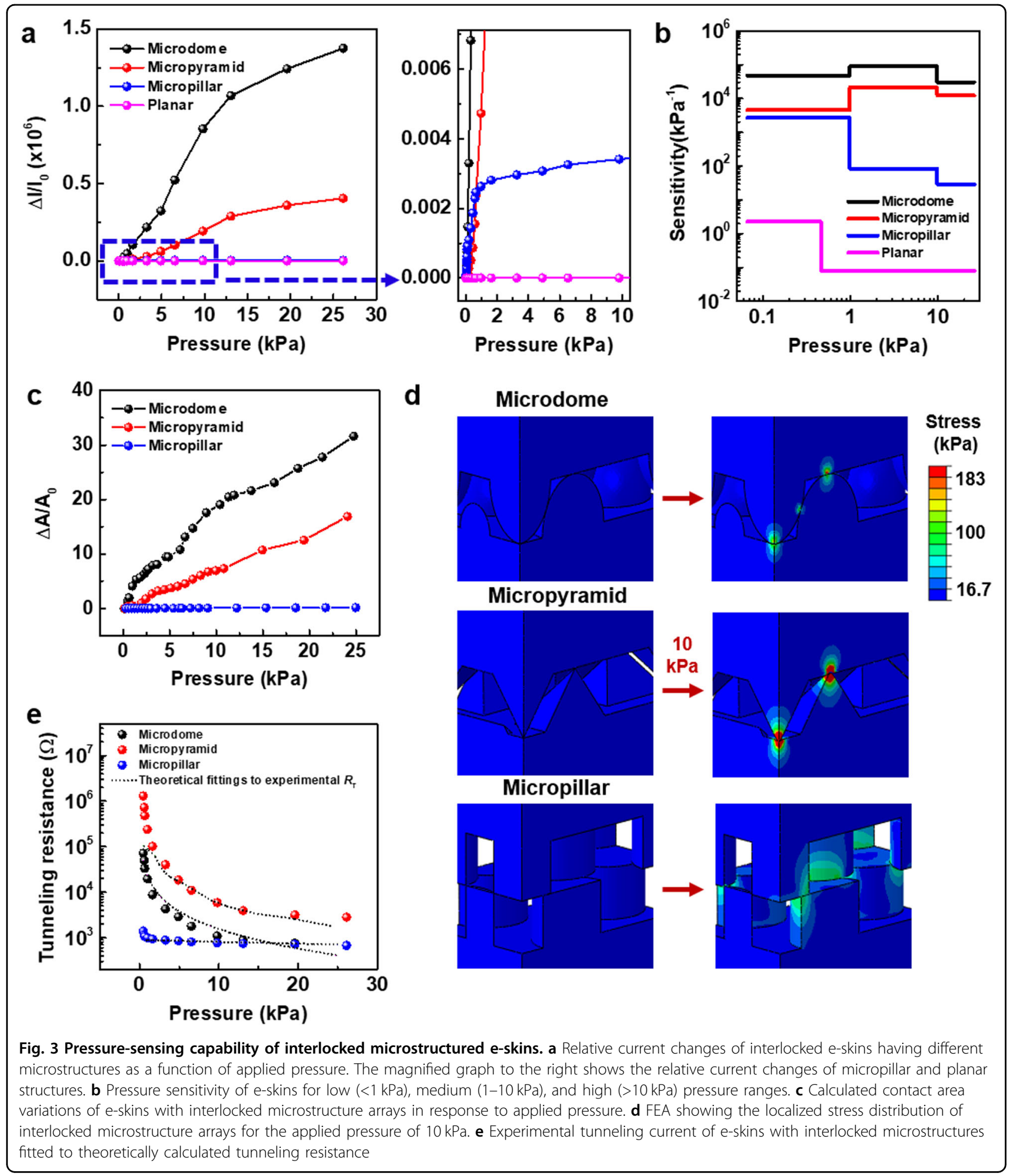

response to pressure and the lowest minimum detection level $(0.09 \mathrm{~Pa}$, corresponding to air flow of $1 \mathrm{~m} / \mathrm{s}$ on $1.5 \times$ $1.5 \mathrm{~cm}^{2}$ of sensor) compared to the other microstructures, indicating the critical role of inter-microdome contacts on the enhanced piezoresistance (Supplementary Fig. S7). To show the possibility of integration with commercially available chip, e-skin was connected to the commercial chip and was detectable signals on application of pressure (Supplementary Fig. S8). To understand the different trend on $\Delta I / I_{0}$ variations depending on microstructure 


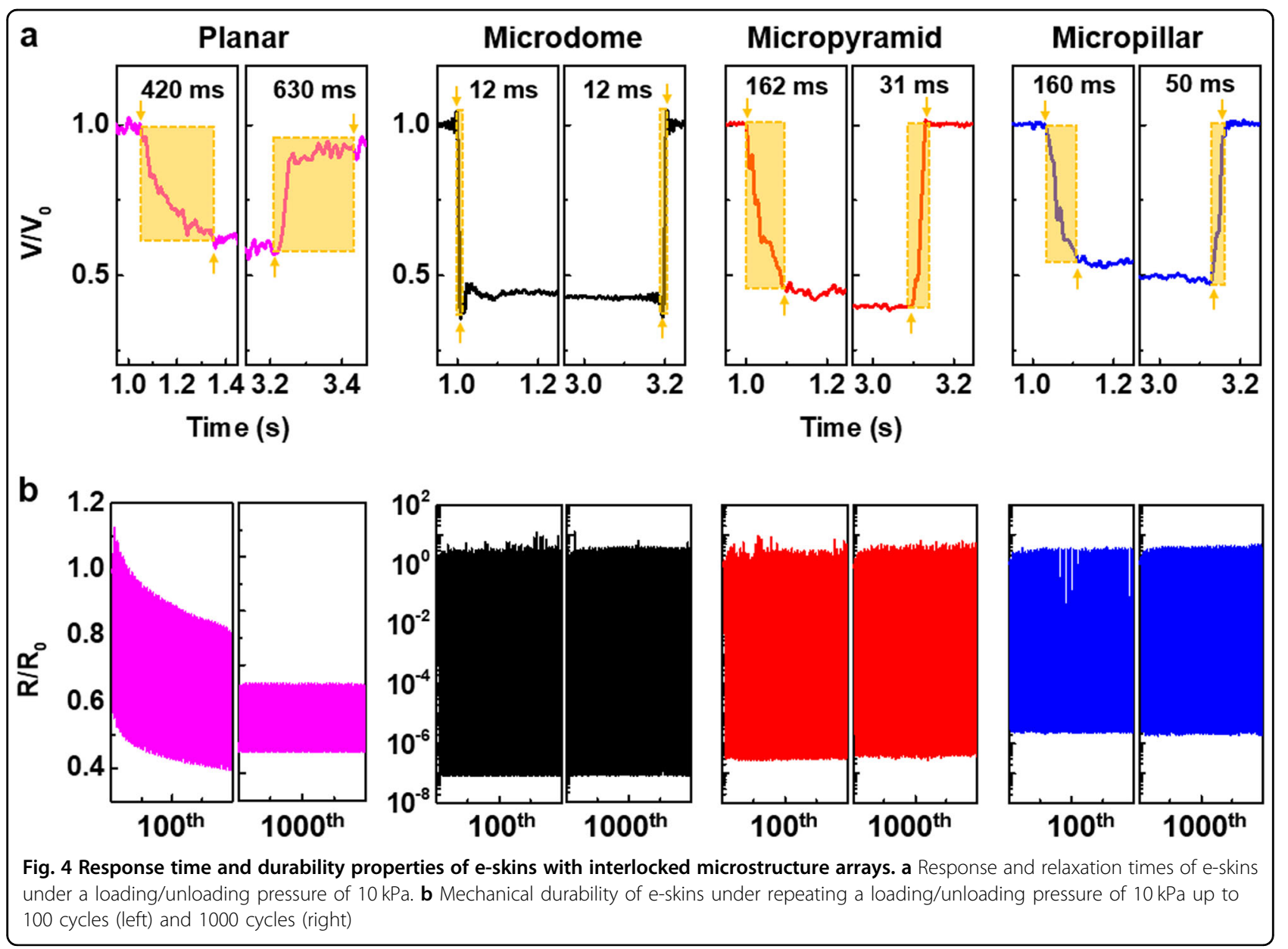

shapes, finite-element simulations of contact-area change under normal pressure were performed. Figure $3 \mathrm{c}$ indicates that the microdome structure exhibited the largest increase in relative contact area $\left(A / A_{0}\right)$ as compared to micropyramid and micropillar arrays (the absolute values are shown in Supplementary Fig. S9), supporting the shape-dependent trend of $\Delta I / I_{0}$ variations in Fig. 3a. This simulation result indicates that the rate of contact-area change with respect to pressure decreased in the following order: dome $>$ pyramid $>$ pillar, which is the same tendency as that for single-sided microstructured films.

To elucidate the sensing performances of interlocked microstructure e-skins, pressure sensitivity was analyzed (Fig. 3b and Supplementary Fig. S10). All of the e-skins having interlocked microdome, micropyramid, and micropillar structures exhibited great pressure sensitivity: $47,062 \mathrm{kPa}^{-1}, 4569 \mathrm{kPa}^{-1}$, and $2733 \mathrm{kPa}^{-1}$ in the lowpressure range $(<1 \mathrm{kPa}) ; 90,657 \mathrm{kPa}^{-1}, 21,308 \mathrm{kPa}^{-1}$, and $85 \mathrm{kPa}^{-1}$ in the medium-pressure range $(1-10 \mathrm{kPa})$; and $30,214 \mathrm{kPa}^{-1}, 12,219 \mathrm{kPa}^{-1}$, and $29 \mathrm{kPa}^{-1}$ in the highpressure range $(>10 \mathrm{kPa})$, respectively. Compared to the planar structure, these microstructures exhibited extremely high pressure sensitivity and a wide dynamic pressure range before saturation. This can be attributed to the larger change in contact area (Fig. 3c) and insulating layer thickness (Supplementary Fig. S11) at the interface of interlocked microstructures. Since the tunneling current of interlocked microstructures primarily depends on variations of contact area and insulating layer thickness, it is worth to compare the theoretical calculation of tunneling current with the experimental one to further understand the working mechanism of piezoresistive interlocked microsctructures. The experimental tunneling resistance can be extracted by using the relation $R_{\mathrm{T}}=R-$ $R_{\mathrm{F}}-R_{\mathrm{CR}}$, where $R$ is the total resistance of interlocked composite film, $R_{\mathrm{F}}$ is the bulk composite film resistance, and $R_{\mathrm{CR}}$ is the constriction resistance. $R_{\mathrm{CR}}$ can be neglected in our system due to the comparable size between the diameter of MWNTs and intertube contact area $^{29}$. The theoretical tunneling resistance $\left(R_{\mathrm{T}}=(V / J) \times\right.$ $\left.\left(1 / A_{\mathrm{CNT}}\right)\right)$ can be estimated by calculating the tunneling current density $(J)$ according to Simmons model ${ }^{33}$ and the actual electrical contact area $\left(A_{\mathrm{CNT}}\right)$. Figure $3 e$ exhibits theoretical tunneling current of different microstructures, 
which are fitted to experimental tunneling current. The calculation details are provided in Supplementary Section 1. The theoretical and experimental results match well, which support that variations of contact area and insulating layer thickness significantly affect the tunneling current and thus the pressure sensitivity.

In particular, the interlocked microdome structures exhibited remarkable sensitivities over broad pressure ranges, which are much better than those of previously reported e-skins (Supplementary Table S1). It is also worth noting that there is a critical difference in sensitivity values depending on whether the sensitivity is expressed in terms of either current $\left(S=\left(\Delta I / I_{0}\right) / \Delta P\right)$ or resistance $\left(S=\left(\Delta R / R_{0}\right) / \Delta P\right)$. In the e-skin system with a drastic variation in electrical response reported in the present study, the sensitivity expressed in terms of current is more adequate for the precise analysis of sensitivity because the relative resistance change becomes $1\left(\Delta R=R-R_{0} \approx R_{0}\right.$ and $\left.\Delta R / R_{0} \approx 1\right)$ and thus underestimates the sensitivity when the initial resistance is significantly higher than the resistance under pressure.

Applications of pressure-sensitive e-skins in robotics and healthcare devices require fast response time and repeated usability. Figure 4a shows the response time of interlocked composites having different microstructures upon loading and unloading of a $10 \mathrm{kPa}$ pressure. The response time of interlocked composites with microdome, micropyramid, and micropillar arrays were 12, 162, and $160 \mathrm{~ms}$, respectively, which were significantly faster than that of planar composite films $(420 \mathrm{~ms})$. The slow piezoresistive response time of planar composites can be attributed to the viscoelastic properties of PDMS, which induces a time delay for bulk film deformation in response to stress. On the other hand, the piezoresistive property of interlocked microstructures is dominated by surface microstructure deformation rather than bulk film deformation, leading to minimization of time-delay behavior. Additionally, sufficient void spaces between the interlocked microstructures enabled a facile release of energy, resulting in faster recovery times than that for the planar structure $^{13}$. In particular, the interlocked microdome arrays exhibited the fastest response time ( 35 times faster than that of planar structures, Fig. 4a) due to the largest variation in contact area (Fig. 3c), which facilitates the immediate formation of a conductive path. Figure $4 \mathrm{~b}$ shows the interlocked microstructures also exhibited excellent mechanical stability over repeated cycles. Piezoresistive e-skins having interlocked microstructures presented uniform piezoresistive properties upon repetitive pressure loadings $(10 \mathrm{kPa})$ over 1000 times without any surface crack (Supplementary Fig. S12). In contrast, eskins having planar structures exhibited non-uniform and unstable piezoresistive properties over repetitive cycles, which can be attributed to incomplete recovery to their original state due to a slow response/relaxation time and a strong stiction between two planar surfaces.

\section{Multidirectional force-sensing capability of interlocked microstructured e-skins}

In human fingertip skin, various mechanoreceptors and interlocked epidermal-dermal ridges enable spatiotemporal recognition of intensity, direction, and location of forces applied in different directions, which is critical for hand-manipulation of objects with different shapes. Artificial e-skins that can detect and discriminate multidirectional forces are required in robotic skins, gesture monitoring devices, and other healthcare sensors. Interlocked microstructures can induce a unique microstructure deformation tendency specific to each microstructure geometry depending on the direction of applied forces. This would result in sensitive and selective piezoresistance in multidirectional force sensing. Figure 5 shows the piezoresistive sensing properties of interlocked microstructures having different geometries in response to shear, tensile, and bending stresses.

Figure 5a shows the shear-force detection properties of interlocked microstructures. In order to engage the upper and lower microstructure layers, a normal preload ( 45 $\mathrm{kPa}$ ) was applied on the upper layer prior to applying shear force. In the linear regime of Fig. $5 \mathrm{a}$, the pillar structure exhibited the highest shear sensitivity $(0.54$ $\mathrm{N}^{-1}$ ), which is $\sim 2$ and $\sim 4$ times larger than that of the dome $\left(0.27 \mathrm{~N}^{-1}\right)$ and pyramid $\left(0.14 \mathrm{~N}^{-1}\right)$ structures. The finite-element simulation results (Supplementary Fig. S13) exhibit that the normal preload induced a microstructure deformation at the contact spots, and the subsequent application of shear force further increased the microstructure deformation in the lateral direction and the contact area between interlocked microstructures. The calculated contact-area change in response to shear force shows that the rate of contact-area variation decreases in the following order: pillar, then dome, and finally the pyramid structure (Fig. 5b), which is the same tendency observed in the experimental results. It is worth noting that the pillar structure exhibited the smaller contact-area change for normal pressure compared to dome and pyramid structures due to the large initial contact area (Fig. 3c). These results imply that once the initial contact is formed between the opposing microstructures under a predefined normal preload, the pillar structure deforms more easily than the other microstructures in response to lateral shear force, which results in the enhanced contact area change and thus improved shear-force sensitivity.

For detecting tensile strain, the micropillar structure exhibited the least sensitive piezoresistance compared to the microdome and micropyramid structures (Fig. 5c). For tensile strain, the output current decreases due to the 


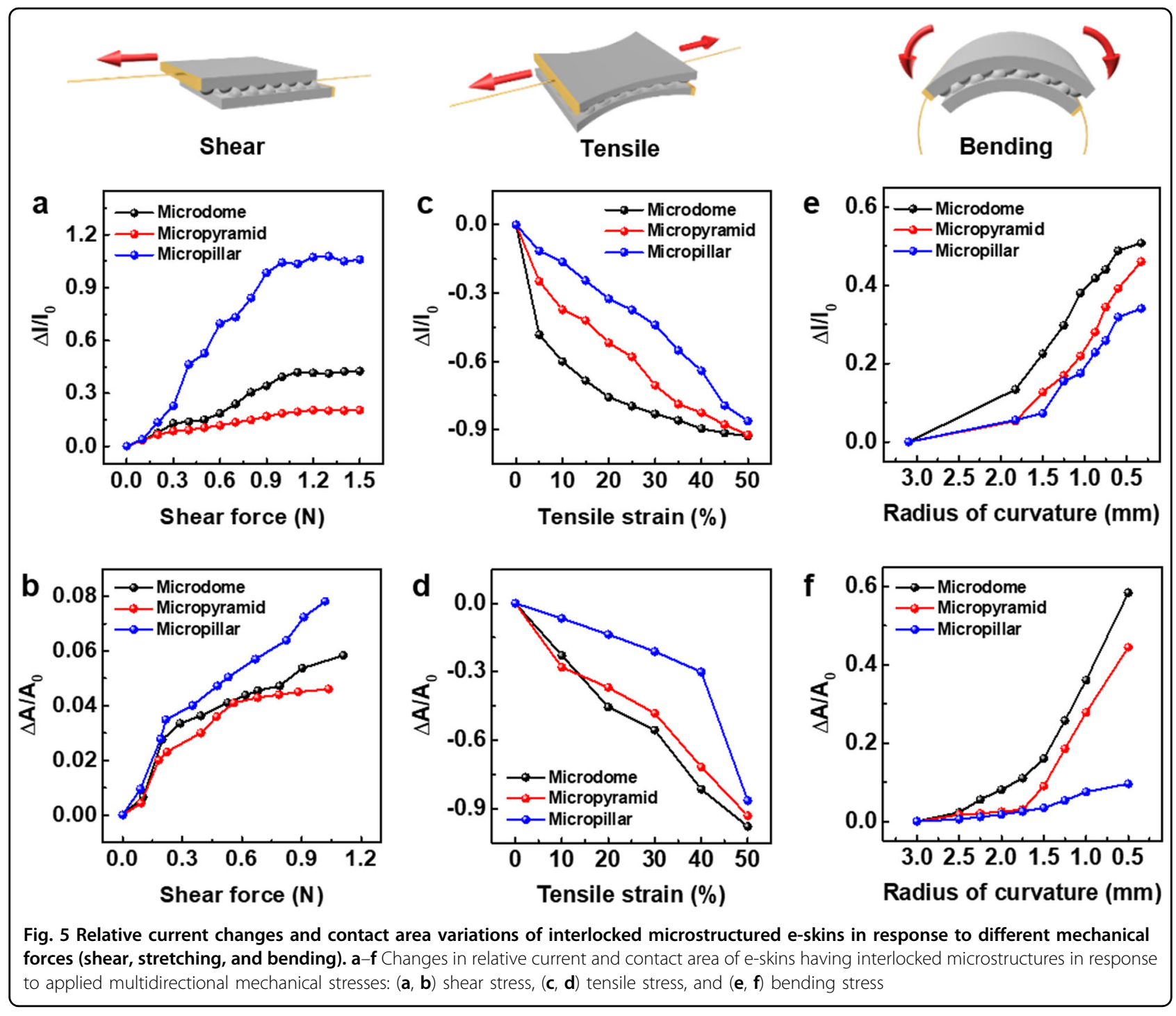

deformation of hexagonal microstructure arrays into elongated hexagonal arrays in the tensile direction, which results in a decreased contact area and thus decreased output current ${ }^{26}$. The simulation results indicate that the lateral tensile strain induced a decrease in concentrated stress at the contact spots and in the contact area between opposing microstructured films (Supplementary Fig. S14), leading to a decrease in output current. For the quantitative comparison of strain-sensing properties, a gauge factor $(G F)$ was analyzed. This is defined by the equation $G F=\left(\Delta R / R_{0}\right) /\left(\Delta L / L_{0}\right)$, where $R$ is the electrical resistance and $L$ is the length of film. The interlocked microdomes exhibited the largest GF ( 16), which was about 2 and 7 times larger than those of the micropyramid $(\sim 7)$ and micropillar ( 2.2) structures for tensile strains up to $30 \%$ (Supplementary Fig. S15). The calculated contact-area change in Fig. 5d also exhibits a similar tendency with the order microdome, then micropyramid, and finally micropillar for the rate of contact area change in response to tensile strain. As a result of bending strain, the concentrated stress and contact area of interlocked microstructures increased with bending curvature (Supplementary Fig. S16). Similar to normal-pressure and tensile-strain sensing, the interlocked microdome structure exhibited the largest bending strain sensitivity, followed by the micropyramid and micropillar structures (Fig. 5e). The calculated contact-area change in Fig. $5 f$ similarly exhibits the largest contact-area change for interlocked microdome structures.

In addition to force sensitivity, discrimination of multidirectional forces is an essential requirement for practical applications of e-skins. In an interlocked geometry of microstructures, distinct deformation patterns depending on the type of mechanical forces induce unique output current signals specific to the applied forces ${ }^{26}$. This geometry-specific force-responsive behavior exhibited by 


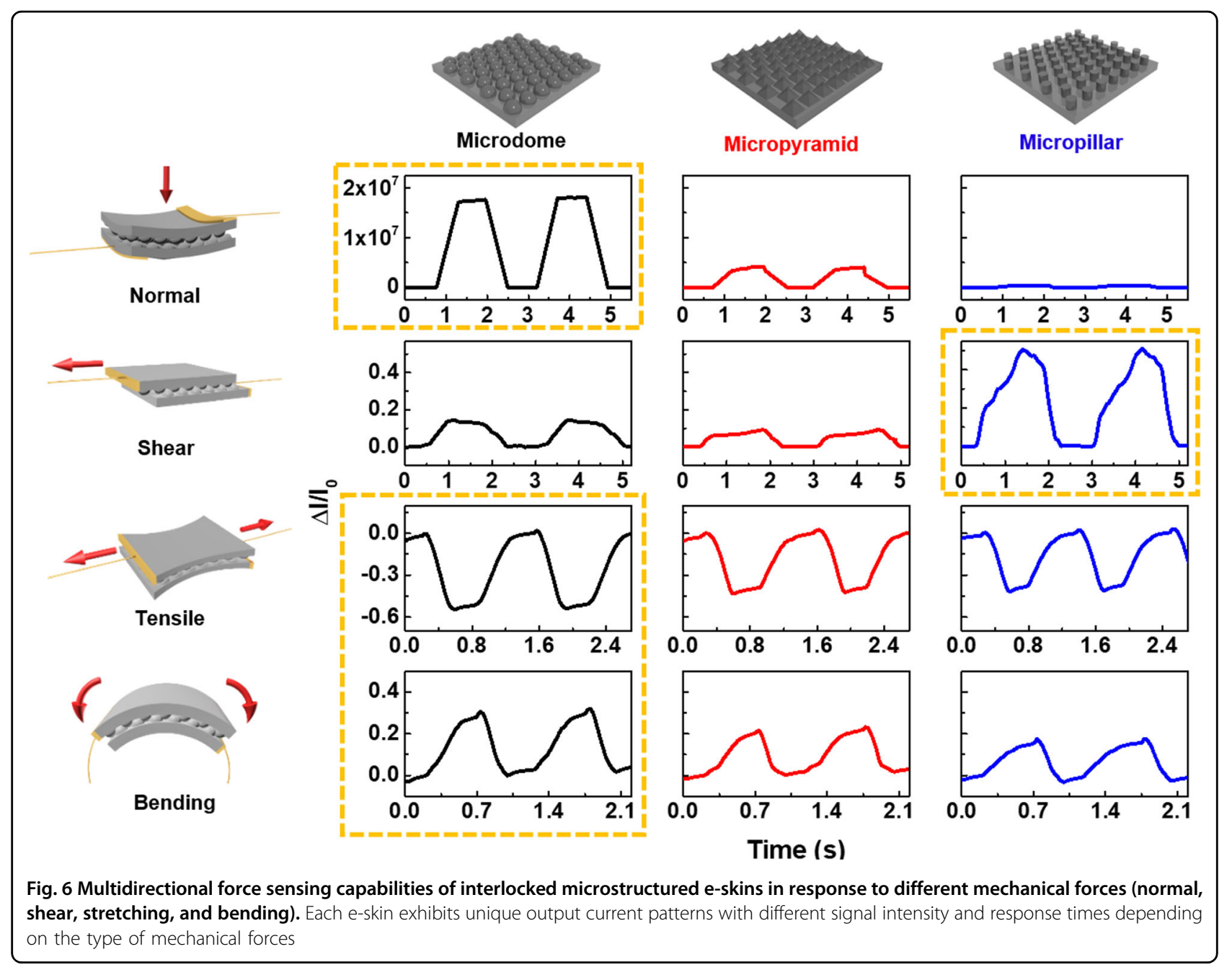

interlocked microstructures enables detection and differentiating of multidirectional mechanical stimuli. Each eskin having different microstructures exhibited unique output current patterns with different signal intensity and response times depending on the type of mechanical forces (normal, shear, tensile, and bending; Fig. 6). It was also observed that differently shaped microstructures exhibited different signal intensities and patterns under the same mechanical stimuli. In particular, microdome structures exhibited the most sensitive piezoresistance for normal pressure, tensile strain, and bending stimuli. In contrast, the micropillar structures showed the most sensitive piezoresistance in response to shear force. All of these different signal patterns are attributed to different magnitudes of deformations of the interlocked microstructures and to response/relaxation times depending on the direction of the applied forces and the shape of the microstructures. Although the microdome structures exhibited the most sensitive piezoresistance for normal pressure, tensile strain, and bending stimuli compared to micropyramid and micropillar structures for the equal microstructures feature sizes $(10 \mu \mathrm{m}$ diameter, $15 \mu \mathrm{m}$ pitch, and $6 \mu \mathrm{m}$ height), it is worth to note that the force sensitivity of e-skins also depends on the feature size such as the height, diameter, and pitch of periodic microstructures in addition to the microstructure shape. For example, as shown in Supplementary Fig. S17, the increase of pillar height of micropillar structures results in the enhanced force sensing capabilities. It is expected that the variation of other feature sizes (diameter, pitch) of microstructures significantly affects the multidirectional force sensitivities, which requires further in-depth and systematic studies.

\section{Healthcare device applications of microstructured e-skins}

For practical applications of the piezoresistive e-skins in healthcare monitoring devices, the detection of various bio-signals was compared over a wide range of pressures, from acoustic wave $(<1 \mathrm{~Pa})$ and human breath $(1 \mathrm{~Pa}-1$ $\mathrm{kPa})$ to human pulse pressure (1-10 $\mathrm{kPa}$, e.g., artery pulse pressure, average $30-40 \mathrm{mmHg}, 4-5.3 \mathrm{kPa}$ ) and carotid pulse pressure (average $35 \mathrm{mmHg}, 4.67 \mathrm{kPa})^{34,35}$. The 


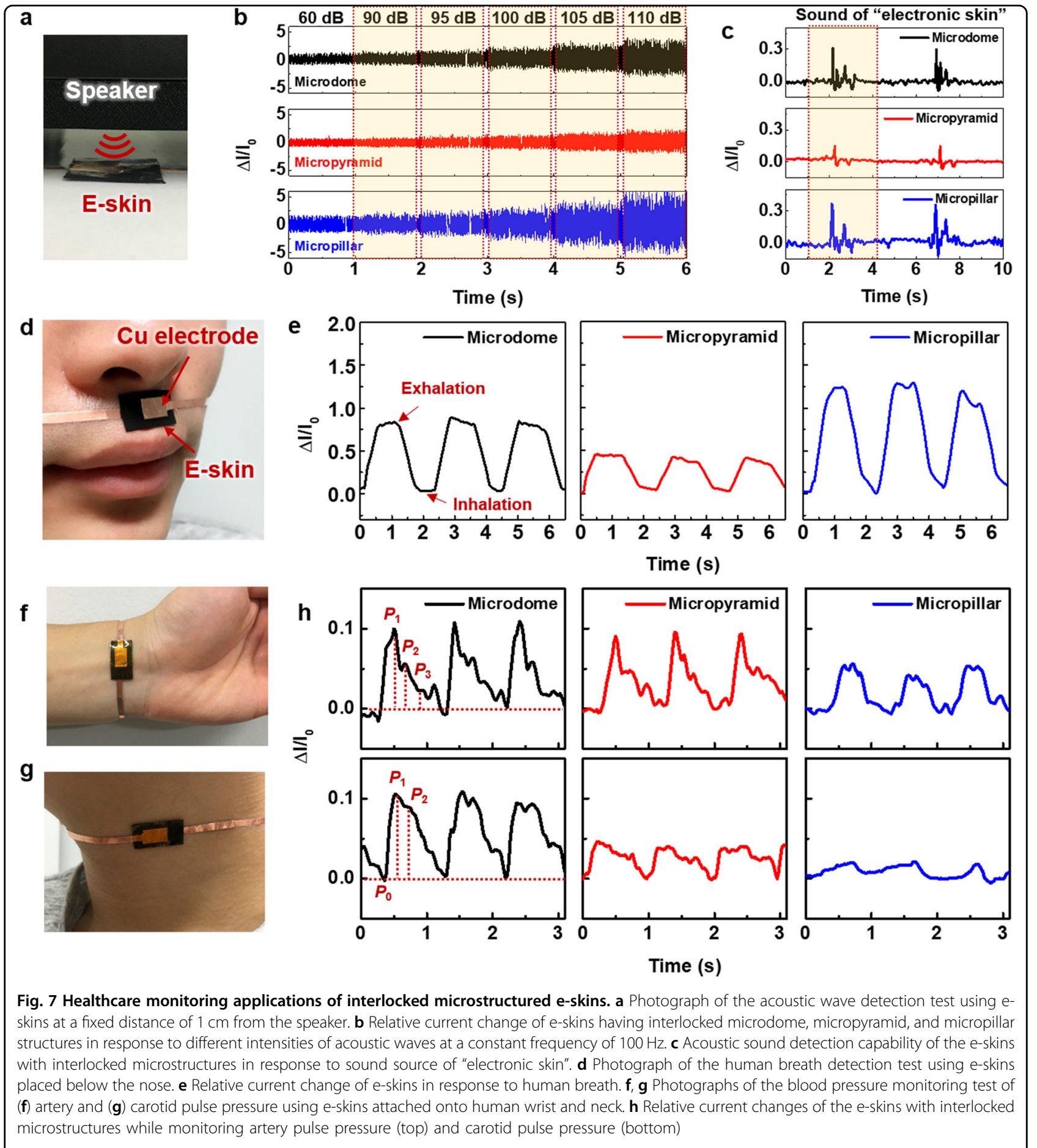

detection of acoustic waves allows for the application of eskins in hearing-aid devices, highly sensitive microphones, and human-machine interfaces. In order to analyze the capability of sound detection, the e-skins were positioned $1 \mathrm{~cm}$ away from the speaker (Fig. 7a) and the output currents were measured in response to acoustic waves with sound pressure levels varying from 90 to $110 \mathrm{~dB}$ at a constant frequency of $100 \mathrm{~Hz}$ (Fig. 7b). As the sound pressure levels increased, the vibration-induced fluctuation of current gradually increased. In particular, the micropillar structure exhibited the best capability to detect acoustic waves, which can be attributed to the 
vibration-induced fluctuation of large initial contact area of interlocked micropillar structures. The detection of acoustic waves at low acoustic pressure and different frequency is also applicable to verify the precise detection of acoustic wave (Supplementary Fig. S18). Figures S18a and S18b show the detection capability of acoustic wave depending on the sound intensity $(60-85 \mathrm{~dB})$ under fixed frequency $(50 \mathrm{~Hz})$ and different frequency $(50-150 \mathrm{~Hz})$ under fixed intensity $(80 \mathrm{~dB})$, respectively. Furthermore, to verify the precise detection, we extracted the detected frequency by a high pass frequency filter $(>65 \mathrm{~Hz}$ ) (Supplementary Fig. S18c) and compared the experimental and theoretical acoustic waves (Supplementary Fig. S18d). The great acoustic wave sensing capabilities of our piezoresistive e-skins are caused by the high pressure sensitivity, which can be favorably compared with the previous acoustic sensors (Supplementary Table S2). Based on the capability to perceive acoustic waves, these e-skins can also recognize the specific words by detecting unique signal patterns with noise filtering process as shown in Fig. 7c. When the e-skins are exposed to sound source of the word "electronic skin" with intensity of $110 \mathrm{~dB}$, different signal patterns can be observed. As expected, the micropillar structure shows the most distinct signal patterns due to the large fluctuation of contact area under tiny vibration. For the detection of human breathing motion, the e-skin was attached right below the nose (Fig. 7d). For repeated breathing motion, all the e-skins exhibited periodical electrical signals corresponding to inspiration/inhalation (Fig. 7e). Particularly, the micropillar structure exhibited the largest change in output current, which can be attributed to the largest shear force sensitivity of micropillar structures, as shown in Fig. 5a. The results also exhibit similar characteristics at low operating-voltages (0.1 and $1 \mathrm{~V}$, Supplementary Fig. S19), where the low operating voltage of $0.1 \mathrm{~V}$ is comparable to that of previously reported e-skins (Supplementary Table S1) and provides a great possibility for low power consumption of wearable devices.

For the detection of artery pulse signals, the output current signals of the e-skins $\left(\sim 1 \mathrm{~cm}^{2}\right)$ were recorded after attaching them onto the wrist (Fig. 7f). All the e-skins having different microstructures showed periodic waveforms of 62 beats $/ \mathrm{min}$ (Fig. 7h), corresponding to the average heartbeat of adult males. An artery pulse waveform contains three distinguishable peaks: $P_{1}$ for the main pulse pressure and $P_{2}$ and $P_{3}$ for the reflected wave pressures from the hand and lower body, respectively ${ }^{36}$. The most distinct pressure peaks were observed for the interlocked microdome structures (Fig. $7 \mathrm{~h}$ ), which is in accordance with the highest pressure-sensitivity of microdome structures in the medium pressure regime (1-10 kPa; Fig. 3a). For a quantitative comparison of artery pulse wave detection capabilities, the radial artery augmentation index $\left(A I_{\mathrm{r}}=P_{2} / P_{1}\right)$ and radial diastolic augmentation $\left(D A I=P_{3} / P_{1}\right)$ were analyzed. The $\mathrm{AI}_{\mathrm{r}}$ and DAI acquired for the microdome and micropyramid structures are 0.53 and 0.19 and 0.54 and 0.2 , respectively; this closely matched the average values of healthy 28 -yearold males ${ }^{36}$. However, the interlocked micropillar structure did not exhibit clearly distinguishable pulse pressure peaks. Furthermore, the carotid pulse pressure was measured by attaching e-skins onto the neck (Fig. 7g). The resulting waveforms presented the distinguished peaks corresponding to the inflection point of the carotid distention pressure $\left(P_{1}\right)$ and common carotid distention pressure $\left(P_{2}\right)$ (Fig. $7 \mathrm{~h}$ ), which provide cardiovascular information based on the pressure augmentation index (PAI), $P A I=\left(P_{2}-P_{1}\right) /\left(P_{2}-P_{0}\right)$. Similar to the results for artery pressure, the interlocked microdome structure exhibited the clearest detection peaks due to high sensitivity at the pulse pressure ranges. The PAI value acquired for the microdome structure was in good accordance with that of healthy 28-year-old males $(\mathrm{PAI}=-3.4)^{37}$.

\section{Conclusions}

We developed multidirectional force sensitive e-skins which possess customizable force sensitivity and selectivity by controlling the specific microstructure geometries (i.e., microdome, micropyramid, and micropillar). Different microstructure shapes of e-skins exhibited distinguishable variations in contact area and localized stress between the two opposing composite films, which critically affect force sensitivity, selectivity, and response/ relaxation times of e-skins for multi-directional (i.e., normal, shear, tensile, and bending) mechanical forces. It was found that microdome structures presented the best force sensitivities for normal, tensile, and bending stresses. For shear stress, micropillar structures exhibited the highest sensitivity. All of the interlocked microstructures exhibited better sensitivities compared to single microstructures and planar films, which can be explained by the finite-element calculation of contact-area change and stress distribution. As proof-of-concept demonstrations, e-skins were successfully employed in the monitoring of various bio-signals over a wide range of pressures, from acoustic wave $(<1 \mathrm{~Pa})$ and human breath $(1 \mathrm{~Pa}-1 \mathrm{kPa})$ to human pulse pressure $(1-10 \mathrm{kPa})$. The findings in this study related to direction-specific sensitivities of e-skins depending on the microstructure shape are a strong precedent for developing high-performance and customizable e-skins for applications requiring directionspecific force-sensing capabilities.

\section{Acknowledgements}

This work was supported by the National Research Foundation of Korea (2015R1A2A1A10054152, 2014M3C1B2048198) and Center for Advanced Soft Electronics under the Global Frontier Research Program (2012M3A6A5055728). 


\section{Author details}

'School of Energy and Chemical Engineering, Ulsan National Institute of Science and Technology (UNIST), Ulsan Metropolitan City 689-798, Republic of Korea. ${ }^{2}$ School of Mechanical and Nuclear Engineering, Ulsan National Institute of Science and Technology (UNIST), Ulsan Metropolitan City 689-798, Republic of Korea. ${ }^{3}$ School of Electrical and Computer Engineering, Ulsan National Institute of Science and Technology (UNIST), Ulsan Metropolitan City 689-798, Republic of Korea

\section{Conflict of interest}

The authors declare that they have no conflict of interest.

\section{Publisher's note}

Springer Nature remains neutral with regard to jurisdictional claims in published maps and institutional affiliations.

Supplementary information accompanies this paper at https://doi.org/ 10.1038/s41427-018-0031-8.

Received: 1 August 2017 Revised: 28 November 2017 Accepted: 4 January 2018.

Published online: 11 April 2018

\section{References}

1. Pang, C. et al. Highly skin-conformal microhairy sensor for pulse signal amplification. Adv. Mater. 27, 634-640 (2015).

2. Boutry, C. M. et al. A sensitive and biodegradable pressure sensor array for cardiovascular monitoring. Adv. Mater. 27, 6954-6961 (2015).

3. Luo, N. et al. Flexible piezoresistive sensor patch enabling ultralow power cuffless blood pressure measurement. Adv. Funct. Mater. 26, 1178-1187 (2016)

4. Jeong, J. W. et al. Capacitive epidermal electronics for electrically safe, longterm electrophysiological measurements. Adv. Healthc. Mater. 3, 642-648 (2014).

5. Lochner, C. M., Khan, Y., Pierre, A. \& Arias, A. C. All-organic optoelectronic sensor for pulse oximetry. Nat. Commun. 5, 5745-5751 (2014).

6. Son, D. et al. Multifunctional wearable devices for diagnosis and therapy of movement disorders. Nat. Nanotech. 9, 397-404 (2014).

7. Liao, $X$. et al. Flexible and highly sensitive strain sensors fabricated by pencil drawn for wearable monitor. Adv. Funct. Mater. 25, 2395-2401 (2015).

8. Liao, X. et al. A highly stretchable ZnO@ fiber-based multifunctional nanosensor for strain/temperature/UV detection. Adv. Funct. Mater. 26, 3074-3081 (2016).

9. Park, J., Lee, Y., Ha, M., Cho, S. \& Ko, H. Micro/nanostructured surfaces for selfpowered and multifunctional electronic skins. J. Mater. Chem. B. 4, 2999-3018 (2016).

10. Bae, G. Y. et al. Linearly and highly pressure-sensitive electronic skin based on a bioinspired hierarchical structural array. Adv. Mater. 28, 5300-5306 (2016).

11. Lee, K. Y. et al. Fully packaged self-powered triboelectric pressure sensor using hemispheres-array. Adv. Energy Mater. 6, 1502566 (2016).

12. Shin, S.-H., Kim, Y.-H., Lee, M. H., Jung, J.-Y. \& Nah, J. Hemispherically aggregated $\mathrm{BaTiO}_{3}$ nanoparticle composite thin film for high performance flexible piezoelectric nanogenerator. ACS Nano 8, 2766-2773 (2014).

13. Mannsfeld, S. C. et al. Highly sensitive flexible pressure sensors with microstructured rubber dielectric layers. Nat. Mater. 9, 859-864 (2010).

14. Choong, C. L. et al. Highly stretchable resistive pressure sensors using a conductive elastomeric composite on a micropyramid array. Adv. Mater. 26, 3451-3458 (2014)
15. Tee, B. C. K. et al. Tunable flexible pressure sensors using microstructured elastomer geometries for intuitive electronics. Adv. Funct. Mater. 24, 5427-5434 (2014)

16. Pang, C. et al. A flexible and highly sensitive strain-gauge sensor using reversible interlocking of nanofibres. Nat. Mater. 11, 795-801 (2012).

17. Park, H. et al. Stretchable array of highly sensitive pressure sensors consisting of polyaniline nanofibers and Au-coated polydimethylsiloxane micropillars. ACS Nano 9, 9974-9985 (2015).

18. Maschmann, M. R. et al. Bioinspired carbon nanotube fuzzy fiber hair sensor for air-flow detection. Adv. Mater. 26, 3230-3234 (2014).

19. Seol, M.-L. et al. Design strategy for a piezoelectric nanogenerator with a wellordered nanoshell array. ACS Nano 7, 10773-10779 (2013).

20. Chou, H.H. et al. A chameleon-inspired stretchable electronic skin with interactive colour changing controlled by tactile sensing. Nat. Commun. $\mathbf{6}$ 8011-8020 (2015).

21. Jang, D. et al. Force-assembled triboelectric nanogenerator with highhumidity-resistant electricity generation using hierarchical surface morphology. Nano Energy 20, 283-293 (2016).

22. Fan, F.-R. et al. Transparent triboelectric nanogenerators and self-powered pressure sensors based on micropatterned plastic films. Nano Lett. 12 3109-3114 (2012)

23. Lee, J.-H. et al. Micropatterned P(VDF-TrFE) film-based piezoelectric nanogenerators for highly sensitive self-powered pressure sensors. Adv. Funct. Mater. 25, 3203-3209 (2015).

24. Deng, W. et al. Microstructure-based interfacial tuning mechanism of capacitive pressure sensors for electronic skin. J. Sens. 2016, 8 (2016).

25. Park, J. et al. Giant tunneling piezoresistance of composite elastomers with interlocked microdome arrays for ultrasensitive and multimodal electronic skins. ACS Nano 8, 4689-4697 (2014).

26. Park, J. et al. Tactile-direction-sensitive and stretchable electronic skins based on human-skin-inspired interlocked microstructures. ACS Nano $\mathbf{8}$ 12020-12029 (2014).

27. Park, J., Kim, M., Lee, $Y$., Lee, H. S. \& Ko, H. Fingertip skin-inspired microstructured ferroelectric skins discriminate static/dynamic pressure and temperature stimuli. Sci. Adv. 1, e1500661 (2015).

28. Park, J. et al. Ultrasensitive piezoresistive pressure sensors based on interlocked micropillar arrays. BioNanoSci 4, 349-355 (2014).

29. Ruschau, G. R., Yoshikawa, S. \& Newnham, R. E. Resistivities of conductive composites. J. Appl. Phys. 72, 953 (1992).

30. Hu, N., Karube, Y., Yan, C., Masuda, Z. \& Fukunaga, H. Tunneling effect in a polymer/carbon nanotube nanocomposite strain sensor. Acta Mater. 56 2929-2936 (2008)

31. Fisher, J. C. \& Giaever, I. Tunneling through thin insulating layers. J. Appl. Phys. 32, 172 (1961).

32. Huang, Z. et al. Pyramid microstructure with single walled carbon nanotubes for flexible and transparent micro-pressure sensor with ultra-high sensitivity. Sens. Actuator A-Phys. 266, 345-351 (2017).

33. Simmons, J. G. Generalized formula for the electric tunnel effect between similar electrodes separated by a thin insulating film. J. Appl. Phys. 34 1793-1803 (1963).

34. Zang, Y., Zhang, F., Di, C.-a. \& Zhu, C. Advances of flexible pressure sensors toward artificial intelligence and health care applications. Mater. Horiz. 2, 140-156 (2015)

35. Mitchell, G. F. et al. Changes in arterial stiffness and wave reflection with advancing age in healthy men and women. Hypertension 43, 1239-1245 (2004).

36. Nichols, W. W. Clinical measurement of arterial stiffness obtained from noninvasive pressure waveforms. Am. J. Hypertens. 18, 3S-10S (2005).

37. Hirata, K., Yaginuma, T., O'Rourke, M. F. \& Kawakami, M. Age-related changes in carotid artery flow and pressure pulses. Stroke 37, 2552-2556 (2006). 